\title{
EDITORIAL
}

\section{Comparison of Echocardiographic Patterns between Mild to Moderate and Severe CKD with type 4 Cardio renal Syndrome Patients}

A good number of patients having Chronic Kidney Diseases are also experiences some sorts of cardiac complaints side by side. This is why we thought that there may be special changes in echocardiographic parameters or structural appearances directing the fingers towards commencement of cardiac disease. And our clinical experiences also shows that changes in cardiac structure and function detected by echocardiography are common in patients with type 4 cardiorenal syndrome. So we designed a protocol to evaluate and compare of echocardiographic patterns in different groups of patients with type 4 cardiorenal syndrome. This comparative observational study was carried out in Cardiology and Nephrology Department of Bangabandhu Sheikh Mujib Medical University, Dhaka, during October 2014 to October 2015 with a total of 100 patients admitted with type 4 cardiorenal syndrome in the above mentioned hospital were included in this study. Patients having mild to moderate CKD (GFR $\geq 30 \mathrm{ml} / \mathrm{min} / 1.73 \mathrm{~m}^{2}$ ) with type 4 cardiorenal syndrome $(\mathrm{n}=56)$ was considered as group I and 44 patients having severe CKD (GFR $<30 \mathrm{ml} / \mathrm{min} / 1.73$ $\left.\mathrm{m}^{2}\right)$ with type 4 cardiorenal syndrome $(\mathrm{n}=44)$ was considered as group II. Patients with liver disease, history of essential hypertension, untreated hypothyroidism, corpulmonale and patients who didn't agree to participate into this research work were excluded from the study. Statistical analyses were carried out by using the Statistical Package for Social Sciences version 20.0 for Windows (SPSS Inc., Chicago, Illinois, USA). P values $<0.05$ was considered as statistically significant. Results shows 10(13.89\%) male patients, 2(9.33\%) female patients and
20(27.78\%) male patients, 4(18.67\%) female patients had LVH in group I and group II respectively. Ischemic heart disease was found $11(19.64 \%)$ and 29(65.9\%) patients, arrhythmias were 2(3.57\%) and 6(13.63\%) patients in group I and group II respectively. Heart failure was found $10(22.72 \%)$ patients in group II and none in group I. In group I, 4(7.1\%) patients had systolic dysfunction (EF $<55 \%$ and FS <25\%), 4(7.1\%) patients had valvular calcification. In group II, 12(27.3\%) patients had systolic dysfunction, 28(63.6\%) patients had valvular calcification. $36.4 \%$ patients had pericardial effusion in group II and not found in group I. These difference between group I and group II were statistically significant $(\mathrm{p}<0.05)$. 32(57.1\%) patients had diastolic dysfunction $(\mathrm{E} / \mathrm{A}<0.8 \&>2)$ in group I and 24(54.5\%) in group II. Thus we may conclude ischemic heart disease, heart failure, arrhythmia, pericardial effusion, valvular calcification, left ventricular hypertrophy and systolic dysfunction are significantly higher in severe CKD with type 4 cardiorenal syndrome.

So proper blood pressure management as well as periodic cardiovascular monitoring with ECG and Doppler Echocardiogram are easy way to trace on-coming cardiac events in patients with CKD.

Sajal K Banerjee, S M Mustafa Zaman, Rajesh K Ghosh, Harisul Hoque

Department of Cardiology, Bangabandhu Sheikh Mujib Medical University, Shahbag, Dhaka 